\title{
The Effect of Small Scale Motion on an Essentially-Nonlinear Dynamo
}

\author{
Benjamin M. Byington ${ }^{1}$, Nicholas H. Brummell ${ }^{1}$ \& Steven M. Tobias ${ }^{2}$ \\ ${ }^{1}$ Department of Applied Mathematics, University of California Santa Cruz, 1156 High St, \\ Santa Cruz, CA 95064 \\ ${ }^{2}$ Department of Applied Mathematics, University of Leeds, Leeds LS2-9JT, UK
}

\begin{abstract}
A dynamo is a process by which fluid motions sustain magnetic fields against dissipative effects. Dynamos occur naturally in many astrophysical systems. Theoretically, we have a much more robust understanding of the generation and maintenance of magnetic fields at the scale of the fluid motions or smaller, than that of magnetic fields at scales much larger than the local velocity. Here, via numerical simulations, we examine one example of an "essentially nonlinear" dynamo mechanism that successfully maintains magnetic field at the largest available scale (the system scale) without cascade to the resistive scale. In particular, we examine whether this new type of dynamo at the system scale is still effective in the presence of other smaller-scale dynamics (turbulence).
\end{abstract}

Keywords. magnetic fields - MHD - turbulence - dynamos.

\section{Results and Discussion}

The dynamo under consideration operates in an "essentially nonlinear" manner, in the sense described in a paper by Cline, Brummell \& Cattaneo, ApJ, 599: 1449 - 1468, 2003. Such dynamos do not have a typical kinematic phase characterized by negligible Lorentz forces and exponentially growing eigenfunctions. Rather, they require a critical initial magnetic field strength to operate, and then the dynamo generating velocity is a function of the magnetic field itself. Figures 1a and 2a show the dynamic evolution of one realization of this type of dynamo. Here, $B_{x}$ is shown as a diagnostic of dynamo activity. After some initial transience, the system relaxes into a nearly periodic steady state, with strong magnetic structures repeatedly created by the velocity shear and rising via a magnetic buoyancy mechanism. Notice that the magnetic field exists on the scale of the system here without cascading to smaller scales, even though the magnetic Reynolds number (based on the shear velocity) is of order $R_{m} \sim 3000$.

Here, we attempt to assess the effects of turbulence at scales much smaller than the magnetic structures on this dynamo, by applying small scale perturbations to the temperature field. Various spectral signatures and time scales have been considered, but here we will discuss only white noise ( $\delta$-correlated in space and time). The system proves to be highly sensitive to the presence of noise, in the sense that very small perturbations are sufficient to kick the solution into a different nonlinear state (Figure $1 \mathrm{~b} \& 2 \mathrm{~b}$ ). Temperature fluctuations of magnitude $5 \times 10^{-7}$ (cf. a mean temperature of order unity) are sufficient to kick the system into a similar but more energetic and chaotic state with a higher frequency of structure creation. The new dynamics remain if the noise is subsequently turned off, indicating that the new state appears to be the more stable of the two.

This new dynamo state however is very robust to the presence of noise. Varying levels of noise were applied to the system, spanning many orders of magnitude (Figure 3), up to 

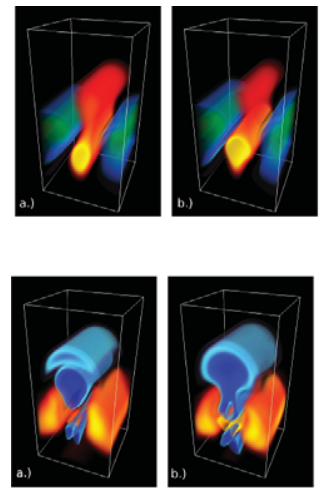
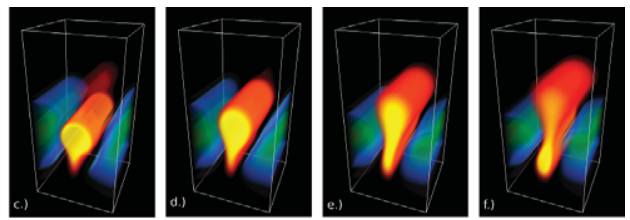

(a) No applied noise
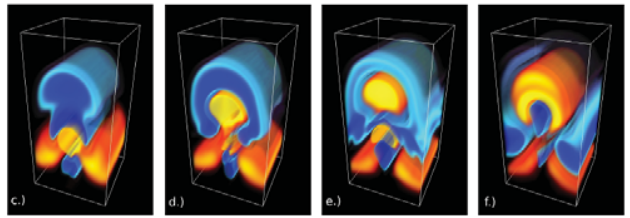

(b) Amp 5e-7 applied noise

Figure 1. Volume Renderings of $B_{x}$ over one period

levels comparable in strength to the background field. Stronger perturbations increases the energy at smaller wavelengths, and increased the level of dissipation at small scales, but even the strongest perturbations failed to disrupt dynamo action.

In conclusion, the system (at these parameters) appears to have two nonlinear states. The first is unstable, giving way to the second with even small applications of noise. However, the second state appears stable and remains even when perturbed by unphysically large amounts of noise. Of particular importance is that we have found a system-scale, essentially nonlinear dynamo that appears to be robust, operating even in the presence of strong small scale perturbations. Future work will examine other parameters and extend the model to include more realistic turbulence instead of ad hoc applications of noise.

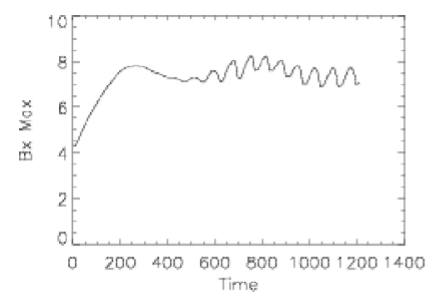

(a) No applied noise

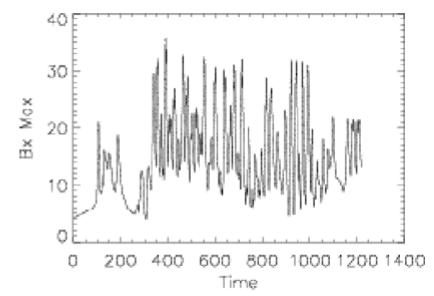

(b) Amp 5e-7 applied noise

Figure 2. Maximal $B_{x}$ for several periods

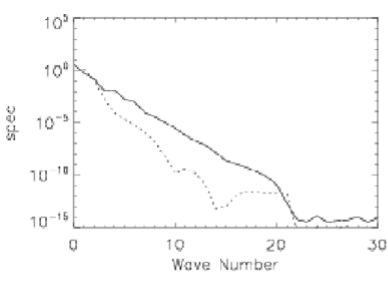

(a) Amp 5e-7

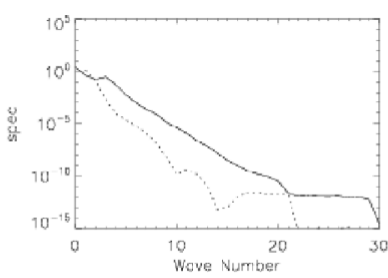

(b) Amp 1e-4

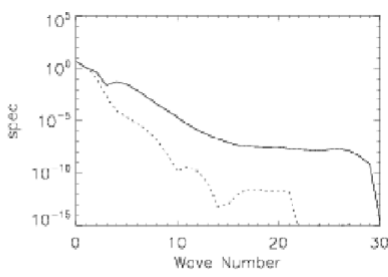

(c) Amp 1e-2

Figure 3. Spectrum of horizontal slice of $B_{x}$ through a typical magnetic structure for several levels of noise. Dashed line: no applied noise. 